\title{
From the $\mathrm{SrTiO}_{3}$ surface to the $\mathrm{LaAlO}_{3} / \mathrm{SrTiO}_{3}$ interface: How thickness is critical
}

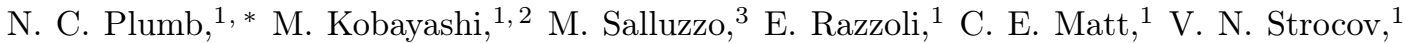 \\ K.-J. Zhou, ${ }^{1}$ C. Monney, ${ }^{1}$ T. Schmitt, ${ }^{1}$ M. Shi, ${ }^{1}$ J. Mesot, ${ }^{1,4}$ L. Patthey, ${ }^{1,5}$ and M. Radovic ${ }^{1,4,5},{ }^{\dagger}$ \\ ${ }^{1}$ Swiss Light Source, Paul Scherrer Institut, CH-5232 Villigen PSI, Switzerland \\ ${ }^{2}$ Department of Applied Chemistry, School of Engineering, University of Tokyo, 7-3-1 \\ ${ }^{3}$ CNR-SPIN, Complesso Universitario Monte S. Angelo, Via Cinthia I-80126 Napoli, Italy \\ ${ }^{4}$ Institut de la Matiere Complexe, EPF Lausanne, CH-1015, Lausanne, Switzerland \\ ${ }^{5}$ SwissFEL, Paul Scherrer Insitut, CH-5232 Villigen PSI, Switzerland
}

\begin{abstract}
Novel properties arising at interfaces between transition metal oxides, particularly the conductivity at the interface of $\mathrm{LaAlO}_{3}(\mathrm{LAO})$ and $\mathrm{SrTiO}_{3}$ (STO) band insulators, have generated new paradigms, challenges, and opportunities in condensed matter physics. Conventional transport measurements have established that intrinsic conductivity appears in LAO/STO interfaces when the LAO film matches or exceeds a critical thickness of 4 unit cells (uc). Recently, a number of experiments raise important questions about the role of the LAO film, the influence of photons, and the effective differences between vacuum/STO and LAO/STO, both above and below the standard critical thickness. Here, using angle-resolved photoemission spectroscopy (ARPES) on in situ prepared samples, as well as resonant inelastic x-ray scattering (RIXS), we study how the metallic STO surface state evolves during the growth of a crystalline LAO film. In all the samples, the character of the conduction bands, their carrier densities, the $\mathrm{Ti}^{3+}$ crystal fields, and the responses to photon irradiation bear strong similarities. However, LAO/STO interfaces exhibit intrinsic instability toward in-plane folding of the Fermi surface at and above the 4-uc thickness threshold. This ordering distinguishes these heterostructures from bare STO and sub-critical-thickness LAO/STO and coincides with the onset of unique properties such as magnetism and built-in conductivity.
\end{abstract}

Unraveling the origins of the electron gases at the STO surface ${ }^{1+3}$ and LAO/STO interfaces ${ }^{4}$, as well as the mechanisms leading to interesting and useful behaviors such as magnetism ${ }^{5} 7$ and persistent photo-enhanced/induced conductivity $\sqrt{819}$, are crucial steps towards fully exploiting the technological promise ${ }^{[0]}$ of these systems. Theories about the existence of the metallic interface in LAO/STO have largely centered around growth-induced defects and doping such as ion intermixing ${ }^{11}$ and oxygen vacancies, as well as the "polar catastrophe" model in which, at the critical thickness of $4 \mathrm{uc} \mathrm{c}^{12}$, carriers transfer across LAO to the STO interface in order to quench the electrostatic potential arising from the growth of polar LAO on nonpolar STO13. While several arguments can be made in favor of the polar catastrophe model ${ }^{14}$, the issue is not yet settled. Meanwhile the origin of interfacial magnetism in LAO/STO remains an open question as well.

A number of recent observations enrich the story surrounding the origins of conductivity at oxide interfaces and surfaces. For instance, interfacial conducting states have been found in other STO-based heterostructures where no polar discontinuity should occur ${ }^{15} \mid 18$. These results come amidst the backdrop of the discovery of a metallic state at the surface of bare STO. Such observations draw attention to the role of the STO substrate and raise the possibility that STO's surface and interface gases are intimately connected; therefore each may be able to teach us about the origins of metallicity and other properties in the other. With this in mind, we set out to investigate the evolution of the low-dimensional metallic state on STO starting from the bare surface and progressing through 4 or more unit cells of LAO over
STO.

We begin our study using RIXS to probe the crystal field excitations in bare STO and a sample with $8 \mathrm{uc}$ of LAO grown over STO. As a "photon in, photon out" technique, RIXS penetrates into the bulk of the sample to probe electron transitions between unoccupied states. By performing RIXS on resonance with $\mathrm{Ti}^{3+}$ absorption features, the technique can be tuned to focus on the surface and interface regions in STO and $\mathrm{LAO} / \mathrm{STO}$, respectively, where the low-dimensional electron gases reside $e^{3 / 19} 22$. Figure 1 a compares RIXS spectra from a bare $\mathrm{TiO}_{2}$-terminated STO substrate and one with an LAO film grown on top ( $8 \mathrm{uc})$. In order to selectively probe the conducting states, we performed RIXS using $h \nu=459.2 \mathrm{eV}$, corresponding to $2 p_{3 / 2}-3 d e_{g}$ absorption by $\mathrm{Ti}^{3+}$ sites. Both samples show a peak near the elastic line at an energy loss of $-110 \mathrm{meV}$ and a weak shoulder at roughly $-200 \mathrm{meV}$, as well as features centered at approximately $-2.5 \mathrm{eV}$ and $-1.7 \mathrm{eV}$. Based on our previous RIXS study of LAO/STO multilayers ${ }^{22}$, we attribute the near-elastic features as intra- $t_{2 g}$ transitions, the structure at $-2.5 \mathrm{eV}$ as $e_{g}-t_{2 g}$ transitions, and the one at $-1.7 \mathrm{eV}$ as arising from delocalized electron excitations. Overall, the spectra clearly demonstrate that cubic symmetry is broken ${ }^{22}$, and the correspondence between these spectral features in both bare STO and LAO/STO is a clear indication that $\mathrm{Ti}^{3+}$ sites in both systems are associated with essentially the same structural distortion - most likely the polar Ti-O buckling seen by several studies of LAO/STO interfaces $23 \mid 24$ and the bare STO surface $^{25}$ 27.

Next we studied STO perturbed under 2 uc of LAO film using in situ ARPES at conventional UV photon en- 
ergies. Even though this thickness of LAO/STO does not have a conducting interface in ambient, dark conditions, scanning tunneling measurements have successfully been performed on such samples when illuminated, implying the existence of conductivity ${ }^{8}$. Moreover, a Fermi surface appears when the sample is irradiated in ultrahigh vacuum (UHV, $\sim 10^{-11}$ mbar). This behavior is similar to how the electron gas found on bare STO can form under analogous conditions $\$ 2 \mid 19$. Fermi surfaces of the bare STO and 2 uc LAO/STO systems obtained using photon energies of $85 \mathrm{eV}$ and $47 \mathrm{eV}$ (corresponding to different cuts through the out-of-plane momentum axis $k_{z}$ ) are shown in Figs. 1 b-e. Figs. 1f-g show side-by-side comparisons of energy-vs.- $k_{x}$ dispersion data from bare STO and 2 uc LAO/STO obtained at $k_{y}=0$ in the first Brillouin zone using $h \nu=85 \mathrm{eV}$. The ellipsoidal components (heavy bands) of the FS extend slightly farther in $k$ than in bare STO (a little over $0.5 \pi / a$ along the long axes from the Brillouin zone center, instead of $\sim 0.4 \pi / a$ for bare STO $\left.{ }^{19}\right)$, qualitatively consistent with calculations ${ }^{28}$. The overall nature of the near- $E_{F}$ electronic structure of the LAO/STO system is similar to the bare surface of STO, whose photon energy dependence in ARPES is attributed to a combination of confined, non-bulk-like quasi-2D and $3 \mathrm{D}$ electronic states associated with $\mathrm{Ti} 3 d_{x y}$ and $\mathrm{Ti}$ $3 d_{x z} / 3 d_{y z}$ bands, respectively 19 . This naturally suggests that the conducting $3 d_{x y}$ and $3 d_{x z} / 3 d_{y z}$ electrons comprising the photo-induced metal in the 2 uc LAO/STO interface region have similar spatial distributions to those in STO. Qualitatively, this result is in agreement with several experimental ${ }^{29 \mid 30}$ and theoretical ${ }^{28 \mid 3132}$ studies of thicker ( $\geq 4 \mathrm{uc}$ ) LAO/STO interfaces, which find that the electron gas actually extends several unit cells into STO. Moreover, the distinct dimensionalities and effective mass characteristics of the $3 d_{x y}$ and $3 d_{x z} / 3 d_{y z}$ electrons naturally explain the observation of two types of carriers in many experiments $5|22| 33 / 34$.

Studying the electronic structure of buried interface states presents a significant challenge for photoemission experiments, since the inelastic mean free path of photoelectrons in a solid is generally considered to be under 1 $\mathrm{nm}$ over a broad range of kinetic energies. Thus we continued in situ ARPES measurements for samples with 4 and 5 uc of LAO by using higher photon energies where resonance enhancement at the $\mathrm{Ti} L$ edge occurs, thereby allowing us to view the momentum-resolved electronic structure associated with Ti.

Figure 2 presents results from resonant angleintegrated photoemission spectroscopy (resonant PES), absorption measured by the total electron yield (TEY), and core level x-ray photoemission spectroscopy (XPS) performed on 5 uc of LAO grown over STO. The sample underwent post-growth annealing in oxygen $\left(P_{\mathrm{O}_{2}}=200\right.$ mbar, $T=55^{\circ} \mathrm{C}, 1 \mathrm{~h}$ ). Over the course of the measurement, exposure to synchrotron radiation causes the spectral characteristics of the LAO/STO sample to evolve in a manner indicating that the metallicity at the interface becomes enhanced. In particular, we observe a transfer of spectral weight within the TEY absorption from $\mathrm{Ti}^{4+}$ to $\mathrm{Ti}^{3+}$ peaks (Fig. 2a), concurrent with a $\mathrm{Ti}^{4+}$-to- $\mathrm{Ti}^{3+}$ redistribution of counts in the $\mathrm{Ti} 2 p$ XPS doublet (Fig. 2b). Complimentary resonant PES data (Fig. 22-e) were obtained by tuning the photon energy over the full Ti $2 p-3 d$ absorption range. The high intensity at the deepest binding energy range of Fig. 2e comes from the $\mathrm{O} 2 p$ valence band, which resonates on both $\mathrm{Ti}^{3+}$ and $\mathrm{Ti}^{4+}$ energies. This demonstrates a noticeable degree of covalency/hybridization in the Ti-O bonds, which may be relevant to theorectical descriptions of the LAO/STO interface. The features that appear at a binding energy of approximately $-1.3 \mathrm{eV}$ come from the "ingap" states previously observed in the case of both STO single crystals ${ }^{2 / 19}$ and LAO/STO heterostructures ${ }^{8 / 35 \mid 36}$, while the spectral weight at the Fermi level indicates that metallicity is present in the sample. Interestingly, the resonance energies of the in-gap states match well with the peak structure of $\mathrm{Ti}^{3+}$ (known from $\mathrm{LaTiO}_{3}{ }^{37 / 38}$ ), whereas states at the Fermi edge resonate slightly off these energies. We speculate that this slight discrepancy might indicate the existence of different varieties of nominal $\mathrm{Ti}^{3+}$ - perhaps located at different depths in the near-interface region and/or with different effective valence $\mathrm{Ti}^{3+\delta(z)}$. Example sketches of resonant photoemission processes corresponding with the level structures of the $\mathrm{Ti}^{4+}$ and $\mathrm{Ti}^{3+}$ states are depicted in Fig. 28. The photon energy ranges where the in-gap and Fermi level states resonate overlap well with the $\mathrm{Ti}^{3+}$ features of the TEY absorption spectrum that become enhanced during photon irradiation. This behavior is very similar to the evolution seen on the surface of bare STO during illumination, where the enhancement of in-gap and metallic states go hand-in-hand 219 .

At this point the similarities between the metallic states seen on the surface of irradiated bare STO and at the $\mathrm{LAO} / \mathrm{STO}$ interface - whether below (2 uc of LAO) or above the standard critical thickness (4-8 uc of LAO) - are striking. All exhibit similar electronic structure, $\mathrm{Ti}^{3+}$ crystal fields, correspondence between the existence of in-gap and metallic electronic states, and creation/enhancement of such states via irradiation. However, the Fermi surfaces of LAO/STO where LAO is at least 4 uc thick show a new characteristic not found in thinner samples or on bare STO. Figure 3 depicts in situ resonant ARPES measurements on bare STO and LAO/STO bilayers with LAO thicknesses of 2, 4, and 5 uc. The data were acquired on resonance with $\mathrm{Ti}^{3+}$ absorption features. Figure 3 a shows Fermi surface mapping on bare STO using resonant and conventional ARPES.

Putting aside fine details concerning the differences between the Fermi surfaces seen by conventional and resonant ARPES, we notice a substantive change occurring at the standard critical LAO thickness. Although all the studied samples show $1 \times 1$ ordering of the LAO surfaces (see Supplementary Information), in those where the thickness of LAO is at least 4 uc (Fig. 3p-d), the 
Fermi surface of the interface shows folding along the $k_{x}$ and $k_{y}$ directions - possibly of a twinned $2 \times 1$ order. Such folding is not seen for either bare STO or 2 uc LAO/STO (Fig. 3a-b). This can also been seen by dispersion cuts along $k_{y}=2 \pi / a$ in the second Brillouin zone for each of the Fermi surface maps (Fig. $3-\mathrm{h}$ ). We observed the same folding pattern (Fig. 4) after annealing the $5 \mathrm{uc} \mathrm{LAO/STO}$ sample in situ under in high oxygen pressure $\left(200\right.$ mbar at $500{ }^{\circ} \mathrm{C}$ for $\left.1 \mathrm{~h}\right)$. This strongly suggests that the in-plane ordering is not directly associated with oxygen vacancies or other defects. With this in mind, the simplest interpretation is that a $2 \times 1$ folding occurs due to twinned rotations of the $\mathrm{TiO}_{6}$ octahedra about the $[100] /[010]$ axes. The proposed rotation pattern is sketched in Fig. 3p, which also qualitatively depicts the ferroelectric Ti-O buckling distortion.

The occurrence of octahedral tilting about $[100] /[010]$ or a similar in-plane reconstruction in sufficiently thick $\mathrm{LAO} / \mathrm{STO}$ interfaces may be connected to unique properties arising in these systems. In particular, various tilting distortions (sometimes combined with ferroelectric Ti-O buckling) in LAO/STO interfaces might correspond with different charge- and/or magnetically-ordered phases, which may even compete in the ground state 39 . Indeed, the onset of the in-plane ordering seen here after depositing 4 or more uc of LAO coincides with the appearance of dilute magnetism in samples studied by scanning superconducting quantum interference device microscopy ${ }^{6}$. Similarly, LAO/STO superlattices were found to exhibit magnetic behavior only when the LAO spacer layers have a minimum thickness of $\sim 4-6 \mathrm{uc}^{7}$.

Concerning the origin of built-in conductivity in $\mathrm{LAO} / \mathrm{STO}$ with at least $4 \mathrm{uc}$ of LAO, it has been shown that the LAO core levels do not exhibit strong thickness dependent shifts or broadening, as would be expected on the basis of the polar catastrophe mode ${ }^{40 / 41}$. Furthermore, hole pockets from LAO that should occur at the Brillouin zone corners are absent in our ARPES data, which is in agreement with an ex situ study 2 . Since the experiments here are performed on samples not exposed to the atmosphere, arguments in favor of an extrinsic passivation of the surface to explain this discrepancy become less likely. Our results also do not favor a direct connection between oxygen vacancies and the built-in conductivity in LAO/STO. The 5 uc LAO/STO sample shows little qualitative change before and after oxygen annealing (Fig. 4), consistent with the idea that oxygen vacancies, while perhaps providing some additional doping, do little to fundamentally alter the interface electron gas 43 . The electronic structure and doping of the metallic state on the surface of bare STO similarly shows no direct link to the concentration of oxygen vacancies ${ }^{1 / 19}$.

While the photoemission experiments do not provide specific confirmation of the origin of the carriers at the interface, they make it clear that distortions in the surface/interface region of STO strongly influence the electronic structure. The polar, ferroelectric-type Ti-O buckling distortion frequently reported on the STO surface and in the interfacial region of STO with LAO is generally thought to play a key role in the spatial confinement of the conducting electrons and compensating the polarity from the LAO film 32144 . Being common to all these systems, Ti-O buckling is likely to account for the shared signatures of symmetry-breaking in the electronic structure: namely the split $3 d_{x y}-3 d_{x z} / 3 d_{y z}$ conduction bands with indications of both $2 \mathrm{D}$ and $3 \mathrm{D}$-like characteristics and the non-cubic crystal field seen in $\mathrm{Ti}^{3+}$ RIXS spectra. The polar buckling region should naturally accommodate

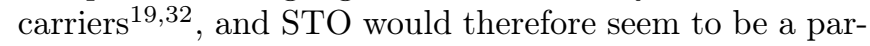
ticularly suitable host for low-dimensional electron gases in large part due to its innate propensity for forming this structure near its surface/interface.

It is interesting to consider how $2 \times 1$ or similar in-plane ordering might be connected to the Ti-O buckling and how, in turn, such ordering might influence the conducting properties of LAO/STO interfaces. Recently, theoretical work has explored the concept of "improper ferroelectricity" in the context of oxide heterostructures, in which ferroelectric displacements occur via coupling to a second order parameter - e.g., various forms of octahedral tilting which are not by themselves polar $\underline{45}$. In principle, even some tilting patterns that in bulk would normally compete with ferroelectric distortions can in certain cases give rise to improper ferroelectricity when the symmetry of the system is broken by an interface $45 \mid 46$. Moreover, it has been proposed that the walls between domains of tilted octahedra (e.g., $2 \times 1$ twin boundaries) in STO may induce polarization $\underline{47}$. Hence the in-plane ordering might strongly influence the nature and/or strength of polar distortions near the interface, which in turn are closely linked to the extent of the confinement region and thus the transport properties.

Multiple effects (e.g., defects, photons, strain) may trigger or otherwise alter the surface/interface structure, thereby affecting the conductivity or other properties. The role of photons in inducing/enhancing the surface and interface conductivity of STO and LAO/STO, respectively, is particularly intriguing. The appearance and/or increase of spectral weight at $E_{F}$ and the enhanced signatures of $\mathrm{Ti}^{3+}$ in the core level and absorption spectra appear to be long-lived; we can move the synchrotron beam away from a heavily exposed spot for hours, then go back, and find the spectral changes largely intact. This is consistent with the persistent photo-induced changes seen in the electronic structure of the STO surface $e^{19}$ and in the transport behavior of LAO/STO and related interfaces ${ }^{9}$. The extremely long lifetimes of the changes imply that photons do more than merely generate carriers by exciting electrons to the conduction band. We therefore propose that light can directly and/or indirectly play some role in altering the structure of the surface/interface region of STO in terms of the polar Ti-O buckling $\frac{19}{19}, 2 \times 1$ or other ordering, and/or the domain structure - all of which could impact the conductivity and spectroscopic properties as discussed above. 
Whatever the origin of the carriers themselves on bare STO and in LAO/STO, the results here emphasize the important role of the near-surface/-interface structure of STO for the realization of a confined electron gas. Indeed, if there is one unifying characteristic of the STO surface and LAO/STO interfaces, it is that conductivity appears to be strongly linked to the existence of ferroelectric Ti-O buckling in the surface/interface region of STO19|23|24|32|48. The similar $\mathrm{Ti}^{3+}$ crystal fields and near- $E_{F}$ electronic structure of bare STO and LAO/STO illustrate the intimate connection between these systems owing to the distortions in their metallic regions. These commonalities, however, belie important differences between the various STO-based systems, and the in-plane ordering seen in the 4- and 5-uc thick LAO/STO interfaces may be an important new insight into how properties such as built-in conductivity and weak/dilute magnetism emerge above the 4-uc threshold.

* nicholas.plumb@psi.ch

† milan.radovic@psi.ch

1 Santander-Syro, A. F. et al. Two-dimensional electron gas with universal subbands at the surface of $\mathrm{SrTiO}_{3}$. Nature 469, 189-193 (2011). URL http://dx.doi.org/10.1038/ nature09720

${ }^{2}$ Meevasana, W. et al. Creation and control of a twodimensional electron liquid at the bare $\mathrm{SrTiO}_{3}$ surface. Nature Mater. 10, 114-118 (2011). URL http://dx.doi. org/10.1038/nmat2943.

${ }^{3}$ Di Capua, R. et al. Observation of a two-dimensional electron gas at the surface of annealed $\mathrm{SrTiO}_{3}$ single crystals by scanning tunneling spectroscopy. Phys. Rev. B 86, 155425 (2012). URL http://link .aps .org/doi/10.1103/ PhysRevB.86.155425

${ }^{4}$ Ohtomo, A. \& Hwang, H. Y. A high-mobility electron gas at the $\mathrm{LaAlO}_{3} / \mathrm{SrTiO}_{3}$ heterointerface. Nature 427, 423-426 (2004). URL http://dx.doi.org/10.1038/ nature02308

${ }^{5}$ Brinkman, A. et al. Magnetic effects at the interface between non-magnetic oxides. Nature Mater. 6, 493-496 (2007). URL http://dx.doi.org/10.1038/nmat1931

${ }^{6}$ Kalisky, B. et al. Critical thickness for ferromagnetism in $\mathrm{LaAlO}_{3} / \mathrm{SrTiO}_{3}$ heterostructures. Nat. Commun. 3, 922 (2012). URL http://dx.doi.org/10.1038/ncomms1931.

7 Salman, Z. et al. Nature of weak magnetism in $\mathrm{SrTiO}_{3} / \mathrm{LaAlO}_{3}$ multilayers. Phys. Rev. Lett. 109, 257207 (2012). URL http://link .aps .org/doi/10.1103/ PhysRevLett.109.257207.

${ }^{\gamma}$ Ristic, Z. et al. Photodoping and in-gap interface states across the metal-insulator transition in $\mathrm{LaAlO}_{3} / \mathrm{SrTiO}_{3}$ heterostructures. Phys. Rev. B 86, 045127 (2012). URL http://link.aps.org/doi/10.1103/PhysRevB.86.

\section{Acknowledgments}

The resonant ARPES and RIXS experiments were performed at the Advanced Resonant Spectroscopies (ADRESS) beamline of the Swiss Light Source (SLS) at the Paul Scherrer Institut in Villigen, Switzerland. The SAXES instrument used for the RIXS experiment was jointly built by the Paul Scherrer Institut and Politecnico di Milano, Italy. The high-resolution ARPES measurements were carried out at the Surface/Interface Spectroscopy (SIS) beamline of the SLS. We are grateful for especially valuable conversations with $\mathrm{H}$. Dil and F. Miletto-Granozio. We also shared informative discussions with R. Claessen, M. Sing, and G. Berner, C. Cancellieri, M. L. Reinle-Schmitt, and C. W. Schneider.

\section{Author contributions}

M. R. conceived the project. N. C. P., M. Salluzzo, E. R., M. K., C. E. M., C. M., K.-J. Z. and M. R. collected data. All authors discussed the results. N. C. P. and M. R. wrote the paper with valuable input from all the coauthors.
045127

${ }^{9}$ di Uccio, U. S. et al. Reversible and Persistent Photoconductivity at the $\mathrm{NdGaO} 3 / \mathrm{SrTiO} 3$ Conducting Interface. ArXiv e-prints (2012). URL http://arxiv.org/abs/ 1206.5083 . 1206.5083.

10 Mannhart, J. \& Schlom, D. G. Oxide interfaces an opportunity for electronics. Science 327, 1607-1611 (2010). URL http://www. sciencemag.org/content/327/ 5973/1607. abstract.

11 Willmott, P. R. et al. Structural basis for the conducting interface between $\mathrm{LaAlO}_{3}$ and $\mathrm{SrTiO}_{3}$. Phys. Rev. Lett. 99, 155502 (2007).

12 Thiel, S., Hammerl, G., Schmehl, A., Schneider, C. W. \& Mannhart, J. Tunable quasi-two-dimensional electron gases in oxide heterostructures. Science 313, 1942-1945 (2006). URL http://www.sciencemag.org/content/313/ 5795/1942. abstract.

13 Nakagawa, N., Hwang, H. Y. \& Muller, D. A. Why some interfaces cannot be sharp. Nature Mater. 5, 204-209 (2006). URL http://dx.doi.org/10.1038/nmat1569

14 Schlom, D. G. \& Mannhart, J. Oxide electronics: Interface takes charge over Si. Nature Mater. 10, 168-169 (2011). URL http://dx.doi .org/10.1038/nmat2965

15 Annadi, A. et al. Unexpected Anisotropic Two Dimensional Electron Gas at the LaAlO3/SrTiO3 (110) Interface. ArXiv e-prints (2012). URL http://arxiv.org/abs/ 1208.6135. 1208.6135.

16 Chen, Y. et al. Metallic and insulating interfaces of amorphous $\mathrm{SrTiO}_{3}$-based oxide heterostructures. Nano Letters 11, 3774-3778 (2011). URL http://pubs.acs .org/doi/abs/10.1021/nl201821j. http://pubs.acs.org/doi/pdf/10.1021/nl201821j. 
17 Chen, Y. Z. et al. A high-mobility two-dimensional electron gas at the spinel/perovskite interface of $\gamma-\mathrm{Al}_{2} \mathrm{O}_{3} / \mathrm{SrTiO}_{3}$. Nat. Commun. 4, 1371 (2013). URL http://dx.doi.org/ $10.1038 /$ ncomms 2394 .

18 Herranz, G., Sánchez, F., Dix, N., Scigaj, M. \& Fontcuberta, J. High mobility conduction at (110) and (111) $\mathrm{LaAlO}_{3} / \mathrm{SrTiO}_{3}$ interfaces. Sci. Rep. 2, 758 (2012). URL http://dx.doi.org/10.1038/srep00758

19 Plumb, N. C. et al. Mixed dimensionality of confined conducting electrons tied to ferroelectric surface distortion on an oxide. ArXiv e-prints (2013). URL http: //arxiv.org/abs/1302.0708, 1302.0708.

${ }^{20}$ Sing, M. et al. Profiling the interface electron gas of $\mathrm{LaAlO}_{3} / \mathrm{SrTiO}_{3}$ heterostructures with hard x-ray photoelectron spectroscopy. Phys. Rev. Lett. 102, 176805 (2009).

21 Cancellieri, C. et al. Interface fermi states of $\mathrm{LaAlO}_{3} / \mathrm{SrTiO}_{3}$ and related heterostructures. Phys. Rev. Lett. 110, 137601 (2013). URL http://link.aps.org/ doi/10.1103/PhysRevLett.110.137601.

${ }^{22}$ Zhou, K.-J. et al. Localized and delocalized Ti $3 d$ carriers in $\mathrm{LaAlO}_{3} / \mathrm{SrTiO}_{3}$ superlattices revealed by resonant inelastic x-ray scattering. Phys. Rev. B 83, 201402(R) (2011).

23 Pauli, S. A. et al. Evolution of the interfacial structure of $\mathrm{LaAlO}_{3}$ on $\mathrm{SrTiO}_{3}$. Phys. Rev. Lett. 106, 036101 (2011). URL http://link .aps .org/doi/10.1103/ PhysRevLett.106.036101

${ }^{24}$ Cantoni, C. et al. Electron transfer and ionic displacements at the origin of the $2 \mathrm{D}$ electron gas at the LAO/STO interface: Direct measurements with atomic-column spatial resolution. Adv. Mater. 24, 3952?-3957 (2012). URL http://dx.doi.org/10.1002/adma.201200667.

25 Bickel, N., Schmidt, G., Heinz, K. \& Müller, K. Ferroelectric relaxation of the $\mathrm{SrTiO}_{3}(100)$ surface. Phys. Rev. Lett. 62, 2009-2011 (1989). URL http://link.aps.org/ doi/10.1103/PhysRevLett.62.2009.

zo Hikita, T., Hanada, T., Kudo, M. \& Kawai, M. Structure and electronic state of the $\mathrm{TiO}_{2}$ and $\mathrm{SrO}$ terminated $\mathrm{SrTiO}_{3}(100)$ surfaces. Surf. Sci. 287-288, 377-381 (1993). URL http://www.sciencedirect.com/science/article/ pii/003960289390806U.

27 Ikeda, A., Nishimura, T., Morishita, T. \& Kido, Y. Surface relaxation and rumpling of $\mathrm{TiO}_{2}$ terminated $\mathrm{SrTiO}_{3}(001)$ determined by medium energy ion scattering. Surf. Sci. 433-435, 520-524 (1999). URL http://www.sciencedirect.com/science/article/ pii/S0039602899000503

${ }^{2}$ Popović, Z. S., Satpathy, S. \& Martin, R. M. Origin of the two-dimensional electron gas carrier density at the $\mathrm{LaAlO}_{3}$ on $\mathrm{SrTiO}_{3}$ interface. Phys. Rev. Lett. 101, 256801 (2008).

29 Copie, O. et al. Towards two-dimensional metallic behavior at $\mathrm{LaAlO}_{3} / \mathrm{SrTiO}_{3}$ interfaces. Phys. Rev. Lett. 102, 216804 (2009). URL http://link . aps .org/doi/10.1103/ PhysRevLett.102.216804

${ }^{30}$ Dubroka, A. et al. Dynamical response and confinement of the electrons at the $\mathrm{LaAlO}_{3} / \mathrm{SrTiO}_{3}$ interface. Phys. Rev. Lett. 104, 156807 (2010). URL http://link.aps. org/doi/10.1103/PhysRevLett.104.156807

${ }^{3}$ Delugas, P. et al. Spontaneous 2-dimensional carrier confinement at the $n$-type $\mathrm{SrTiO}_{3} / \mathrm{LaAlO}_{3}$ interface. Phys. Rev. Lett. 106, 166807 (2011).

32 Stengel, M. First-principles modeling of electrostatically doped perovskite systems. Phys. Rev. Lett. 106, 136803 (2011). URL http://link .aps .org/doi/10.1103/
PhysRevLett.106.136803.

35 Savoia, A. et al. Polar catastrophe and electronic reconstructions at the $\mathrm{LaAlO}_{3} / \mathrm{SrTiO}_{3}$ interface: Evidence from optical second harmonic generation. Phys. Rev. B 80, 075110 (2009). URL http://link .aps .org/doi/10.1103/ PhysRevB.80.075110.

34 Ben Shalom, M., Ron, A., Palevski, A. \& Dagan, Y. Shubnikov-De Haas oscillations in $\mathrm{SrTiO}_{3} / \mathrm{LaAlO}_{3}$ interface. Phys. Rev. Lett. 105, 206401 (2010). URL http: //link.aps.org/doi/10.1103/PhysRevLett.105.206401

35 Koitzsch, A. et al. In-gap electronic structure of $\mathrm{LaAlO}_{3}$ $\mathrm{SrTiO}_{3}$ heterointerfaces investigated by soft x-ray spectroscopy. Phys. Rev. B 84, 245121 (2011).

36 Drera, G. et al. Spectroscopic evidence of in-gap states at the $\mathrm{SrTiO}_{3} / \mathrm{LaAlO}_{3}$ ultrathin interfaces. Appl. Phys. Lett. 98, 052907 (2011). URL http://link.aip.org/ link/?APL/98/052907/1.

37 Abbate, M. et al. Soft-x-ray-absorption studies of the location of extra charges induced by substitution in controlled-valence materials. Phys. Rev. B 44, 54195422 (1991). URL http://link.aps.org/doi/10.1103/ PhysRevB.44.5419.

38 Salluzzo, M. et al. Orbital reconstruction and the twodimensional electron gas at the $\mathrm{LaAlO}_{3} / \mathrm{SrTiO}_{3}$ interface. Phys. Rev. Lett. 102, 166804 (2009).

39 Zhong, Z. \& Kelly, P. J. Electronic-structure-induced reconstruction and magnetic ordering at the $\mathrm{LaAlO}_{3} \mid \mathrm{SrTiO}_{3}$ interface. Europhys. Lett. 84, 27001 (2008). URL http: //stacks. iop.org/0295-5075/84/i=2/a=27001

40 Segal, Y., Ngai, J. H., Reiner, J. W., Walker, F. J. \& Ahn, C. H. X-ray photoemission studies of the metal-insulator transition in $\mathrm{LaAlO}_{3} / \mathrm{SrTiO}_{3}$ structures grown by molecular beam epitaxy. Phys. Rev. B 80, 241107 (2009). URL http://link .aps .org/doi/10.1103/ PhysRevB.80.241107.

41 Slooten, E. et al. Hard x-ray photoemission and density functional theory study of the internal electric field in $\mathrm{SrTiO} 3 / \mathrm{LaAlO} 3$ oxide heterostructures. ArXiv eprints (2013). URL http://arxiv.org/abs/1301.2179. 1301.2179.

42 Berner, G. et al. Direct k-space mapping of the electronic structure in an oxide-oxide interface. ArXiv eprints (2013). URL http://arxiv.org/abs/1301.2824. 1301.2824 .

43 Basletic, M. et al. Mapping the spatial distribution of charge carriers in $\mathrm{LaAlO}_{3} / \mathrm{SrTiO}_{3}$ heterostructures. $\mathrm{Na}$ ture Mater. 7, 621-625 (2008). URL http://dx.doi.org/ $10.1038 /$ nmat2223.

44 Schwingenschlögl, U. \& Schuster, C. Interface relaxation and electrostatic charge depletion in the oxide heterostructure $\mathrm{LaAlO}_{3} / \mathrm{SrTiO}_{3}$. Europhys. Lett. 86, 27005 (2009). URL http://stacks.iop.org/0295-5075/86/i= $2 / a=27005$

45 Stengel, M., Fennie, C. J. \& Ghosez, P. Electrical properties of improper ferroelectrics from first principles. Phys. Rev. B 86, 094112 (2012). URL http://link.aps.org/ doi/10.1103/PhysRevB.86.094112.

40 Bousquet, E. et al. Improper ferroelectricity in perovskite oxide artificial superlattices. Nature 452, 732-736 (2008). URL http://dx.doi .org/10.1038/nature06817

47 Morozovska, A. N., Eliseev, E. A., Glinchuk, M. D., Chen, L.-Q. \& Gopalan, V. Interfacial polarization and pyroelectricity in antiferrodistortive structures induced by a flexoelectric effect and rotostriction. Phys. Rev. B 85, 
094107 (2012). URL http://link.aps.org/doi/10.1103/ PhysRevB.85.094107.
48 Salluzzo, M. et al. Structural and electronic reconstructions at the $\mathrm{LaAlO}_{3} / \mathrm{SrTiO}_{3}$ interface. Adv. Mater. (2013). URL http://dx.doi.org/10.1002/adma.201204555 

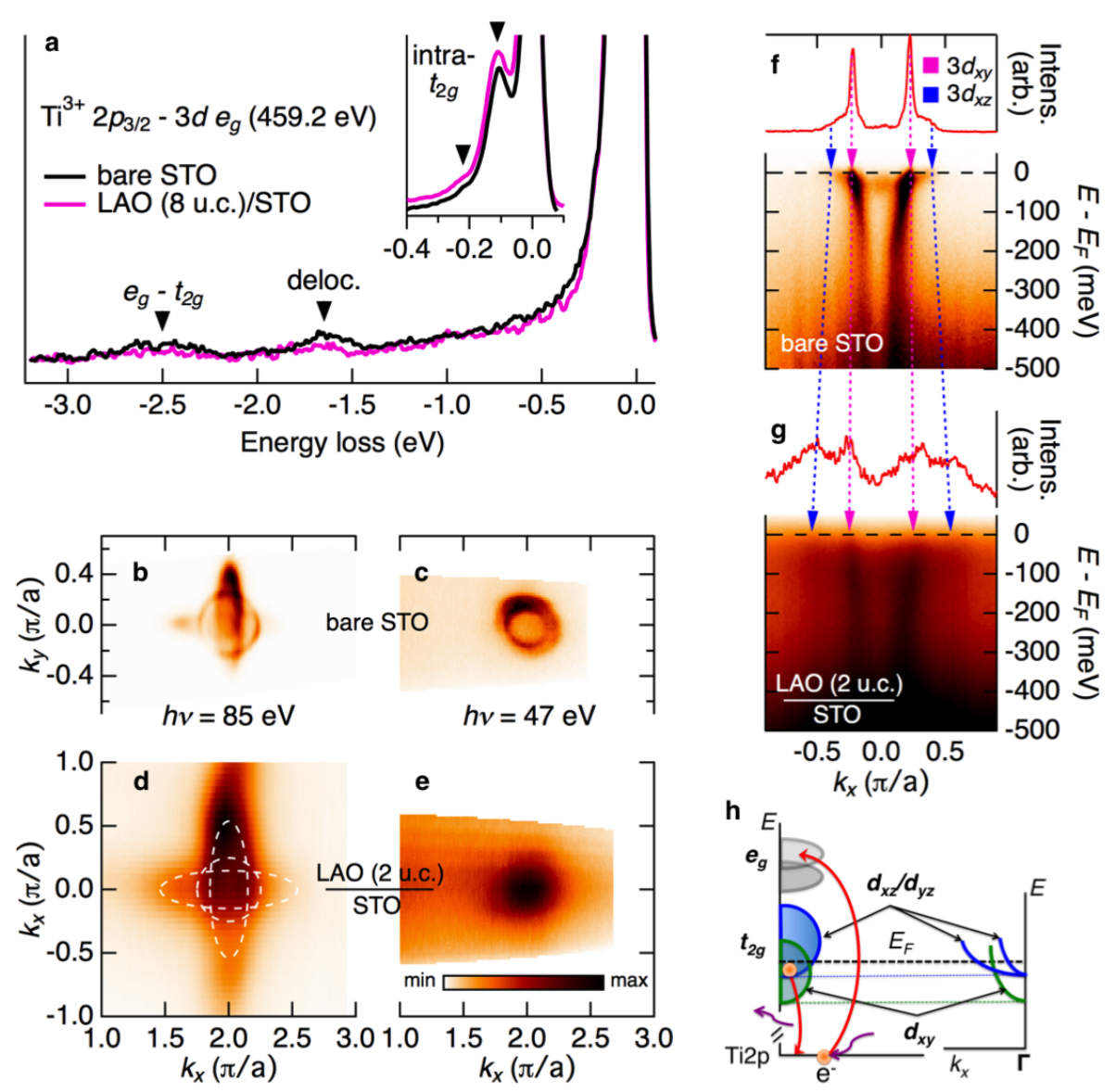

Figure 1 | Comparisons of bare STO and LAO/STO. a, RIXS spectra of STO and LAO/STO. The LAO layer is 8 uc thick. The $e_{g}-t_{2 g} \mathrm{e}$, delocalized electronic, and intra- $t_{2 g}$ transitions (inset) are highlighted. b-e, Conventional ARPES measurements of the Fermi surface of STO under 2 uc of LAO and bare STO at $47 \mathrm{eV}$ and $85 \mathrm{eV}$ in the second Brillouin zone. d, e, Dispersion cuts in the first Brillouin zone through at $k_{y}=0$ for bare STO and STO with 2 unit cells of LAO deposited on top. The photon energy was $85 \mathrm{eV}$. The line profiles show the spectral weight at $E_{F}$, and the markers indicate the Fermi momenta of the outer $3 d_{x y}$ ring (magenta) and $3 d_{x z}$ (blue) bands. The color scales have been enhanced to emphasize key features. The sketched Fermi surfaces in d are based on the Fermi momenta as judged from e. f, Schematic views of the intra- $t_{2 g}$ RIXS process and the corresponding band structure which agrees with the ARPES data. 

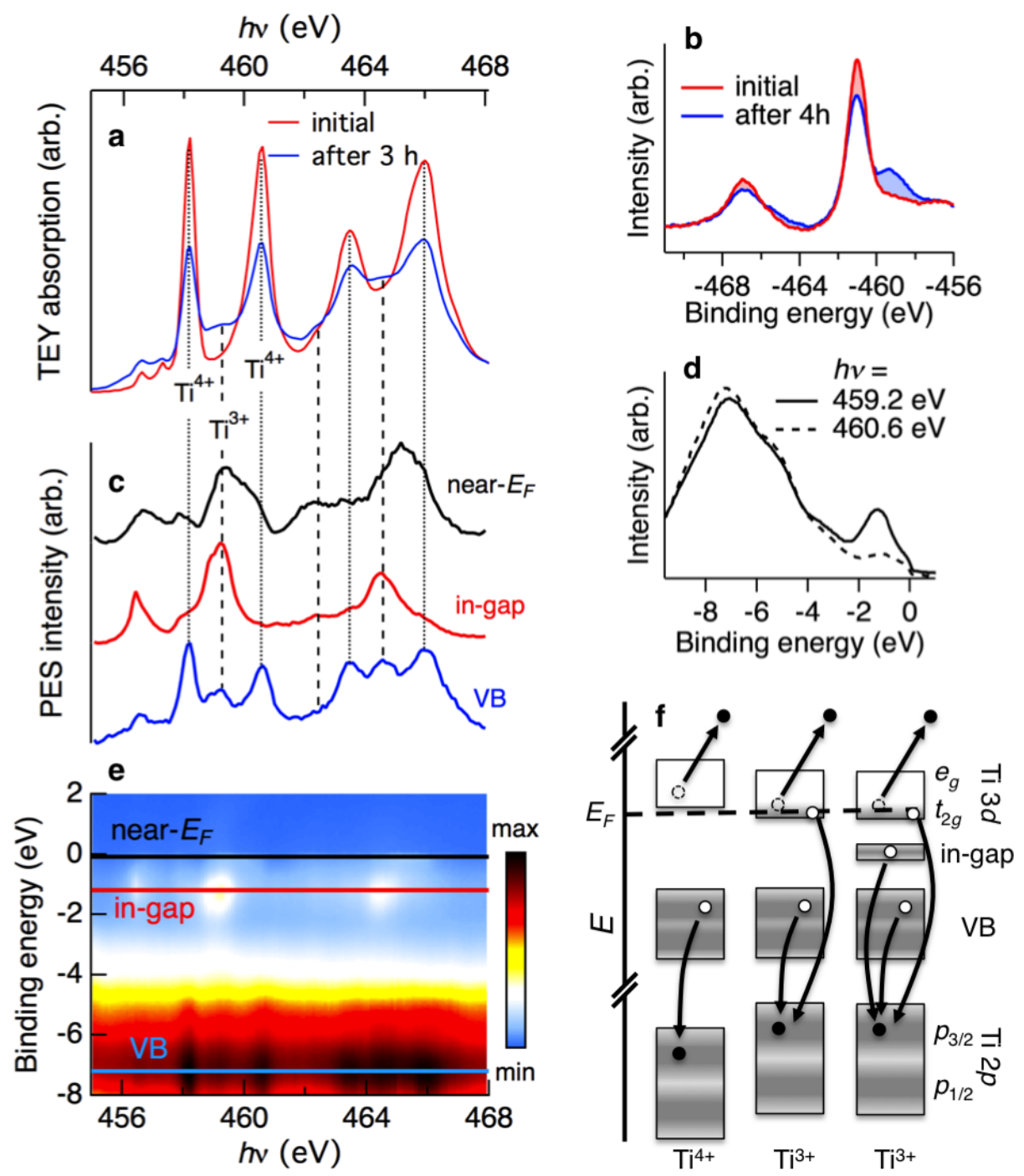

Figure 2 | Resonant photoemission spectroscopy of the interface of $\mathbf{5}$ uc LAO grown over STO. a, Absorption spectra measured by total electron yield (TEY) at an initial time and after 3 hours of exposure to synchrotron radiation under the experimental conditions. b, Ti $2 p$ XPS measured at an initial time and after 4 hours of irradiation. c, Cuts the of angle-integrated PES intensity vs. $h \nu$ at binding energies of $-0.1 \mathrm{eV}$ (near- $\left.E_{F}\right),-1.3 \mathrm{eV}$ (in-gap), and in the valence band at $-7.1 \mathrm{eV}$ (VB). d, Comparison of angle-integrated PES data at $h \nu=460.6 \mathrm{eV}$ (a Ti ${ }^{4+}$ resonance) and $h \nu=459.2 \mathrm{eV}\left(\mathrm{a} \mathrm{Ti}^{3+}\right.$ resonance). e, Map of the PES signal as a function of the incoming photon energy. $\mathbf{f}$, Schematic of resonant photoemission decay channels in the $\mathrm{Ti}^{4+}$ and $\mathrm{Ti}^{3+}$ environments. 

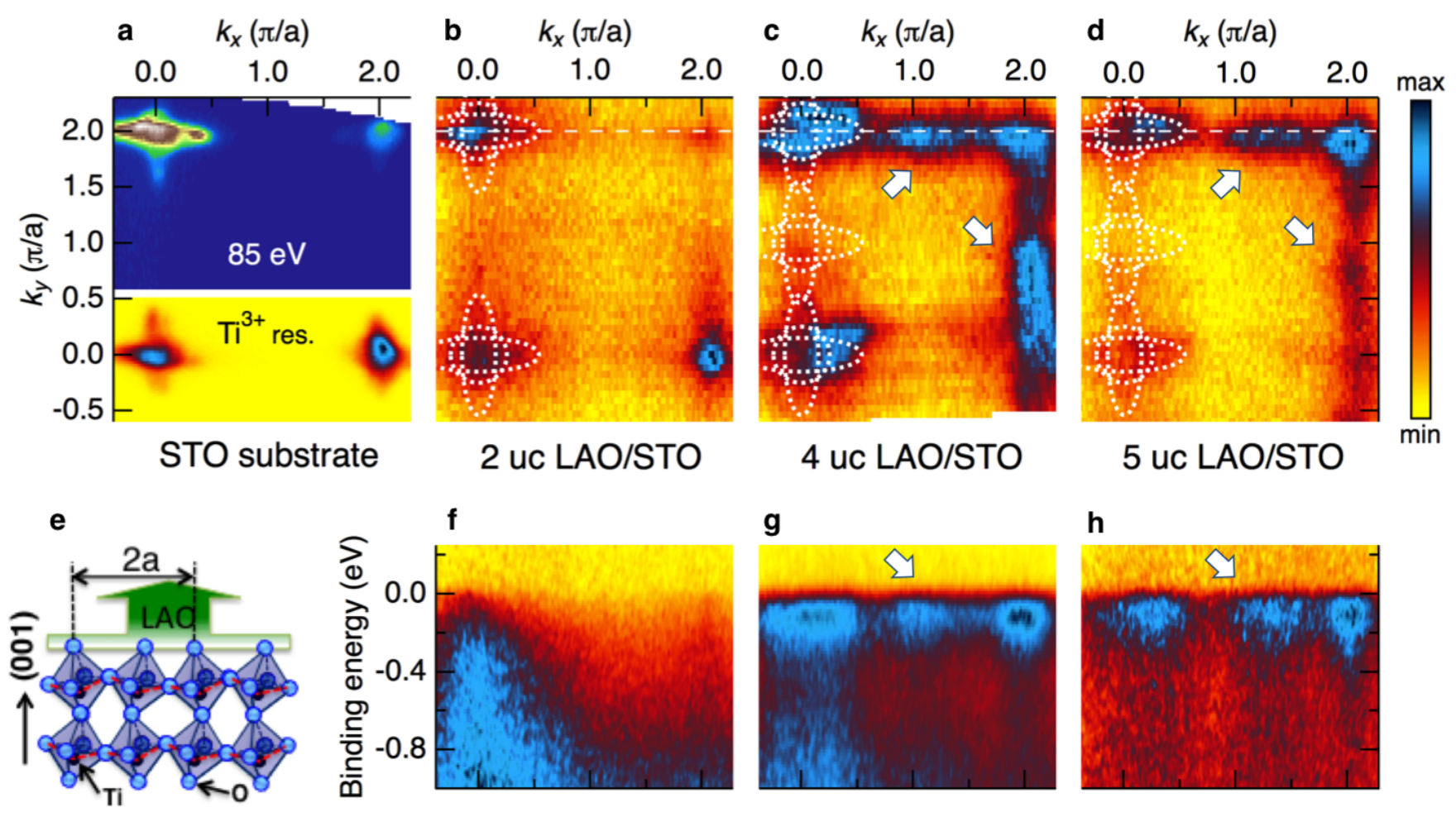

Figure 3 | Evolution of the Fermi surface on STO during deposition of LAO. a, Fermi surface obtained from the surface of a bare STO substrate. The upper map presents data in the $\left(k_{x}, k_{y}\right)=(0, \pi / a)$ and $(\pi / a, \pi / a)$ Brillouin zones obtained by conventional UV ARPES at $h \nu=85 \mathrm{eV}$. The bottom map shows analogous high energy resonant ARPES data obtained using performed on a $\mathrm{Ti}^{3+}$ resonance in the $(0,0)$ and $(0, \pi / a)$ zones. b. Fermi surface of the interface of 2 uc LAO deposited over STO mapped by resonant ARPES. c, Interface of 4 uc LAO over STO. d, Interface of 5 uc LAO over STO. The sketched Fermi surfaces are based on the measurement of 2 uc LAO/STO in Fig. 1 . $\mathbf{f}-\mathbf{h}, E-\mathrm{vs} .-k_{x}$ cuts along $k_{y}=2 \pi / a$ for each of the above maps (b-d). When LAO is deposited above 4 uc, signs of an in-plane reconstruction, marked with arrows, are visible. e, Schematic view of $\mathrm{TiO}_{6}$ octahedral rotations that give rise to $2 \times 1$ ordering in the $x-y$ plane. We probed multiple $\mathrm{Ti}^{3+}$ resonances to confirm the findings; shown here, e.g., are $h \nu=465.3 \mathrm{eV}(\mathbf{a}), 462.6 \mathrm{eV}(\mathbf{b}, \mathbf{d})$, and $459.2 \mathrm{eV}(\mathbf{c})$.
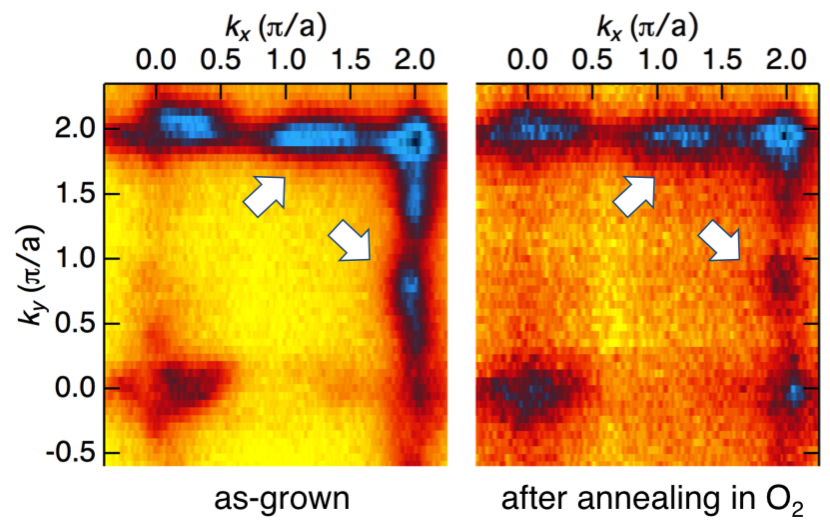

Figure 4 | Comparison of Fermi surfaces of LAO/STO before (left) and after (right) annealing in oxygen atmosphere. The data were obtained using $h \nu=465.3 \mathrm{eV}$. The arrows point to signatures of an in-plane reconstruction. 